\title{
SANTIAGO RAMÓN Y CAJAL: \\ ANÁLISIS RETROSPECTIVO DE UN CASO DE ACOSO ESCOLAR
}

\author{
SANTIAGO RAMÓN Y CAJAL: RETROSPECTIVE \\ ANALYSIS OF A CASE OF SCHOOL HARASSMENT
}

\author{
VICTORIA DEL-BARRIO ${ }^{1}$
}

Cómo referenciar este artículo/How to reference this article:

Del-Barrio, V. (2017). Santiago Ramón y Cajal: análisis retrospectivo de un caso de acoso escolar [Santiago Ramón y Cajal: Retrospective analysis of a case of school harassment]. Acción Psicológica, 14(1), 153-160. http://dx.doi.org/10.5944/ap.14.1.18856

\begin{abstract}
Resumen
El acoso escolar tiene hoy una vigencia especial, sin embargo, es un problema que con mayor o menor intensidad ha existido siempre. Se presenta un caso especial de acoso que por la relevancia del personaje puede ser una fuente de inspiración para los casos actuales de acoso. El método seguido ha sido analizar, primero las características del acoso recurriendo a expertos y segundo las alusiones que Cajal hace del acoso en su obra especialmente el contenido Recuerdos de mi vida donde se hace una minuciosa descripción de su experiencia. Después se comparan ambas situaciones.
\end{abstract}

En la autobiografía de Santiago Ramón y Cajal, se contienen unas descripciones de ciertos episodios de su niñez que resultan ser unas perfectas definiciones de unos casos de "bullying" vividos por él durante sus años de escolar. Esas páginas tienen además el interés de ofrecer unos agudos análisis sobre las causas de aquellos acosos, y sobre su significado para la vida del niño. Dada la procedencia de estos análisis y soluciones tan diversas en distintas circunstancias, es conveniente que los expertos en acoso escolar las conozcan y tengan en cuenta.

Palabras clave: acoso escolar; Ramón y Cajal.

Correspondencia: Victoria del Barrio Gandara. Universidad Nacional de Educación a Distancia (UNED), Calle Juan del Rosal, 14. Madrid. Email: vbarrio@psi.uned.es

ORCID: Victoria del Barrio (http://orcid.org/0000-0003-1629-9011).

${ }^{1}$ Universidad Nacional de Educación a Distancia (UNED), España.

Recibido: 5 de marzo de 2017.

Aceptado: 25 de abril de 2017. 


\begin{abstract}
The school harassment has today a special interest, nevertheless it is a problem that with major or minor intensity has existed always. The followed method has been to analyze, first the characteristics of the bullying appealing experts and I do again the allusions that Cajal does of the bullying in his work specially the content Recuerdos de mi vida where a meticulous description does to itself of his experience. Later both situations are compared. We present a special case of bullying that for the relevancy of the personage can be a font of inspiration for the current cases of bullying In S. Ramón y Cajal's autobiography, there are offered some lively descriptions of a case of violence in school, or bullying, suffered by him during his schoolboy days. His analysis and reflections on that experience may serve as some "classic pages" on such phenomenon, and his way of facing it is still now worthy to be read and considered. Given the origin of these analyses and so diverse solutions in different circumstances, is suitable that the experts in bullying know it and bear in mind.
\end{abstract}

Keywords: bullying; Ramón y Cajal.

\section{Introducción}

La actualidad del tema del acoso escolar en nuestros días se debe a dos causas. Una es el incremento de este tipo de conducta en los últimos diez años; otra, la creciente preocupación social que genera la constante presencia de esos casos en la prensa.

El acoso escolar existe, existió y existirá porque es algo que está intrínsecamente unido al comportamiento humano: la dominación del otro. La sociología, la psicología social y la biología han estudiado copiosamente el hecho de la jerarquización de los individuos que se da en muchas especies, así como los métodos más o menos contundentes que se emplean para alcanzarla.

Una organización social eficaz implica la existencia de un líder y unos seguidores. En términos generales, el líder, por su potencia, habilidad o inteligencia puede encontrar más rápidamente la solución a los problemas con que se enfrenta el grupo y, en tales casos, la mayoría de los miembros reconoce su superioridad y acepta su dirección. Sin embargo, este orden natural se pervierte en cuanto alguien que tiene fuerza se impone a los demás, usándola, pretendiendo ser el líder sin serlo funcionalmente y, sobre todo, recurriendo a la fuerza para lograrlo.

Eso es precisamente lo que ocurre en los casos de acoso (bullying) que hallamos en la escuela: los fuertes se imponen violentamente a los débiles, a los solitarios, a los que son diferentes o tienen algún defecto, aunque sean cosas aparentemente tan nimias como la miopía o la obesidad.

El tema del bullying ha alcanzado altas cotas de atención desde los años setenta. En buena medida el comienzo de su estudio ha sido mérito de D. Olweus (1973), un gran estudioso sueco del acoso infantil. Lo definió como: "un tipo de hostigamiento o agresividad social que de forma reiterada se dirige a determinados menores, denominados como víctimas, y normalmente se produce en el contexto escolar" (Olweus y Hodgins, 1993). A partir de sus investigaciones, muchos estudiosos se han ocupado de este tema en todo el mundo. En España se comenzó a estudiar a finales de los años ochenta (Ortega et al., 1989) y el primer trabajo más conocido es el hecho por el Defensor del Pueblo (1999), con un seguimiento posterior (2007) y secundado por otros muchos trabajos, como iremos viendo.

La violencia en la escuela es muy plural y afecta a la totalidad de estamentos que conviven en ella. Hay violencia de: maestros contra alumnos, de padres contra profesores, de padres contra alumnos, de alumnos contra maestros, y de alumnos contra compañeros. Todas suponen un abuso de poder. Nos ocuparemos aquí sólo de este último, porque es el que guarda relación expresa con el caso de acoso escolar que sufrió Ramón y Cajal en sus días de escolar varias veces.

Notemos que para que se pueda diagnosticar una conducta como acoso o bullying deben darse una serie de condiciones: un desequilibrio de fuerza, poder o número; su carácter reiterativo (2-3 veces al mes, según Olweus, 1993); también debe ser una conducta intimidatoria e intencionada. Si no se dan tales condiciones no se puede 
hablar de acoso, sino, a lo más, de casos de violencia y peleas circunstanciales. A esa conducta cotinuada de hostigamiento se la llama también "violencia escolar" (Garaigordobil y Oñederra, 2010)

Existen muchos grados y tipos de acoso: el físico de gravedad menor: zancadillas, empujones, cachetes, amenazas, pero también el de gravedad mayor: robos, palizas, vejaciones, destrucción de pertenencias. Hay también el acoso psicológico que consiste en críticas de los aspectos intelectuales, morales y sociales del individuo acosado y especialmente de su familia. También existe otro subtipo que es el menosprecio mediante insultos, burlas, bromas, motes o rumores. Otra modalidad es el ostracismo, que consiste en someter al otro al aislamiento. Aunque pudiera parecer que el acoso psicológico no es tan grave como el físico, sus consecuencias negativas son, a menudo, muy negativas y duraderas.

Pero además de todas esas formas activas, se da también lo que se considera un acoso por omisión cuando no se produce ayuda al sujeto que padece acoso (Díaz Aguado, 2016). Es el caso de los espectadores que observan el acoso, lo consienten e incluso jalean o admiran al acosador. La permisividad ante el acoso por parte de los compañeros no acosadores que contemplan la acción, es quizás una de las características sorprendentes del comportamiento de quienes serían las únicas personas que podrían parar este tipo de conductas sin demasiado esfuerzo.

Se considera que el acoso en general y en cualquiera de sus formas puede afectar a un 25 o $30 \%$, de la población escolar; el acoso físico, entre el 3 y $5 \%$ de la misma, y los últimos datos españoles que hoy se tienen apuntan a un incremento de estas cifras en un $75 \%$ (Fundación Anar, 2016). Un reciente informe de la Comunidad de Madrid (2016) compara las denuncias de acoso habidas en 2014-2015, que fueron 69, y las que se registraron en el curso 2015-2016, que llegaron a 179; de ellos 133 fueron en colegios públicos, 43 en concertados $\mathrm{y}$ tres en privados. Estos datos confirman la existencia de un incremento, pero, su ubicación según tipo de enseñanza da solo datos brutos, que habría que completar con unos porcentajes que representasen el valor relativo de tales estimaciones.
Por otro lado, a medida que los niños maduran la adaptación social y el desarrollo mental producen un descenso de la agresión infantil (Tremblay y Nagin, 2005), pero respecto del acoso, por el contrario, se advierte un incremento de dicha conducta a medida que asciende la edad y alcanza su punto máximo en la adolescencia (Garaigordobil, Martinez y Alirin, 2015).

\section{Método}

\section{Evaluación del caso}

El método de evaluación es la observación de la conducta a través de los escritos de Ramón y Cajal que constan en las referencias recogiendo todas las alusiones que narra de sus dificultades en los distintos centros escolares en donde curso sus estudios escolares y bachillerato. La recopilación de datos principalmente se centra en el análisis de su obra: Ramón y Cajal, S. (1923). Recuerdos de mi vida, $3^{\mathrm{a}}$ ed. Madrid: Juan Pueyo y de todas aquellas donde se hace alusión a su vida: Ramón y Cajal, S. (1894), Consideraciones generales sobre la morfología de la célula nerviosa, La veterinaria Española, XXXVIII, Ramón y Cajal, S (1922). Charlas de café. Madrid: Espasa Calpe), Ramón y Cajal, S (1940). Reglas y consejos sobre la investigación científica. (Los tónicos de la voluntad). Madrid: Librería Beltran (8 ed.).

Además, se ha utilizado la definición de acoso escolar de los expertos en la materia Olweus, Diaz Aguado, Fundación Anar: Insultos, agresión física, de una manera repetitiva perpetada por agresores superiores en número y/o edad.

Análisis del caso del acoso en la escolarización de Santiago Ramón y Cajal

Con todos los datos anteriores que conceptualizan y definen el acoso, vamos a contemplar el caso de la borrascosa escolarización de Santiago Ramón y Cajal y analizaremos si se dan en él o no las condiciones de un efectivo acoso escolar. Para empezar por el principio, Ramón y Cajal pertenecía a una familia de clase media baja en el que el padre, D. Justo Ramón, era barbero, que 
con su continuo esfuerzo pasó a ser "cirujano de segunda clase", y, ya en 1858, doctor en medicina y cirugía (Lopez Piñero, 1985). La madre, Antonia, hija de un tejedor, fue toda su vida ama de casa. Vivían en un pequeño pueblecito, Petilla de Aragón, lugar administrativamente navarro, aunque enclavado en Aragón, en donde Cajal nació en 1852. Dos años después se trasladaron a Larrés y a Luna (1852-1856) y después a Valpalmas (1856-60) en donde comenzó su escolarización sin incidentes. D. Justo cambiaba a menudo de pueblo buscando mejoras en el ejercicio de su profesión. El padre de D. Santiago era descrito por su hijo de esta manera:

"Tenaz, hábil, mente vigorosa, potente memoria, trabajador esforzado, austero, creyente en la fuerza de la voluntad. Excelente comadrón de inteligencias".

Este último rasgo se refería sin duda a sus dotes de docente que aplicó duramente sobre su hijo. Este le temía y le admiraba a la vez, y no era para menos, ya que este modesto barbero-cirujano de pueblo que llegó a ser médico consiguió enseñar en la Facultad de Medicina de Zaragoza. A pesar de las diferencias que muy pronto surgieron entre ambos la visión que ofrece de su padre es muy positiva

Curiosamente a su madre, Antonia, la describe a través de las palabras de otros como si temiese no ser ecuánime si la describiese directamente y dice de ella:

"Hermosa, buena, laboriosa. Consentidora a espaldas del padre".

Es cierto que la madre ocultaba a D. Justo, que era muy estricto, algunas de las travesuras de su hijo, Santiago, y le daba de comer a éste a escondidas cuando su padre le castigaba con un ayuno. Muy probablemente esto exigía un gran coraje, porque aquel marido era realmente temible. Su hijo habla a menudo del miedo que le inspiraba su padre al par que admiración.

También se describe a sí mismo como niño:

"Leía y escribía a los 6 años. Era inquieto, travieso, curioso, tímido, antipático, apasionado por la naturaleza y el dibujo".
El que leyera y escribiera a esa corta edad, y más en aquella época, hace pensar dos cosas. Primero, que su padre le instruyó con gran diligencia, puesto que a la edad en que entonces se entraba a la escuela, para aprender allí a leer y a escribir, él ya sabía hacerlo por anticipado. Y, segundo, que era efectivamente un muchacho no sólo inteligente, sino realmente precoz. Cajal se ha referido en otras ocasiones a la inteligencia haciendo hincapié en lo importante que es el esfuerzo mental en la expansión de las conexiones neurales que están a la base de la conducta inteligente (1894). También se puede pensar que su visión de sí mismo era bastante certera, porque toda su biografía posterior vino a respaldar esta primera apreciación de sí. El dibujo, que tan prodigiosas consecuencias tuvo para sus estudios del sistema nervioso y de las innumerables formas de neuronas, le acarreo de niño innumerables problemas con su padre, que trató de inhibir cuanto pudo esa afición, porque no le parecía seria. Dejado a su ser, Cajal hubiese sido pintor, y probablemente muy bueno. Sin embargo, él canalizó esa vocación, frustrada por su padre, hacia el camino de su profesión médica. A su padre le admiraban los estupendos dibujos de huesos que hacía para sus clases de anatomía y más tarde asombraría al mundo con sus dibujos sobre cuanto veía y examinaba con el microscopio.

Más tarde, el periplo familiar les llevó a Ayerbe (1860-1861) y finalmente a Huesca (1861) y a Zaragoza.

En la escuela de Ayerbe fue donde se produjo el primer episodio de acoso. D. Santiago lo narra así:

"Mi aparición en la plaza de Ayerbe fue saludada por una rechifla general de los chicos. De burlas pasaron a las veras. En cuanto se reunían algunos y creían asegurada su impunidad, me insultaban, me golpeaban a puñetazos o me acribillaban a pedradas" (Ramón y Cajal, 1923, p. 23).

Fue un acoso psíquico y físico en toda regla. Los motivos fueron los habituales en este tipo de eventos: llegar nuevo, ser distinto, hablar distinto, vestir distinto:

"Yo no gastaba calzones o alpargatas, ni ceñia con un pañuelo mi cabeza, y eso bastó para que entre aquellos zafios pasara por señorito... Contribuía también mi antipatía y la extrañeza causada por mi lenguaje (Ramón y Cajal, 1923, p. 24)" 
Es curioso que Cajal analiza objetivamente la cuestión y descubre lo que parecen ser las causas usuales del acoso (novedad, vestido y lenguaje) y además añade otra, más bien debida a un defecto de si mismo (antipatía).

A continuación, describe cómo reflexionó e ideó una solución al problema que, en este caso concreto, consistió en asimilarse a las formas de comportarse de los otros:

"Como no era cosa que todos aprendieran el lenguaje de uno, acabé por acomodarme a su extraña jerigonza. Tomé parte en juegos del peón, del tejo, de la espandiella, del marro, carreras, luchas y saltos. Al principio puse resistencia a los juegos brutales, pero el espiritu de imitación pudo más que los sabios consejos de mis padres (Ramón y Cajal, 1923, p. 24). Esto me acarreó fama de pícaro y travieso, pero yo era un infeliz" (Ramón y Cajal, 1923, p. 28).

En este caso optó por una sencilla solución: siguió la regla conocida de "a donde fueres haz lo que vieres", para lograr la adaptación social que le librara del rechazo y el acoso de sus compañeros. Cambió su lenguaje, su modo de hablar, de jugar y de resolver problemas. Pronto se convirtió en el líder del grupo y capitaneaba unas travesuras sin cuento. $\mathrm{Y}$ todo esto lo logró reflexionando, sin pedir auxilio y con solo ocho años, cosa que hace pensar que no sólo era genial mirando por el microscopio. Lo cuenta desde la perspectiva adulta, pero aquellas determinaciones y las acciones las llevó a cabo siendo un completo niño. Cajal (1922) se refiere ya de adulto a las pillerías infantiles como reminiscencias de comportamientos ancestrales orientados a la solución de problemas elementales que presenta la vida (caza, pesca, huida, lucha etc.).

Esta experiencia le dio fama de travieso e indisciplinado. Laín Entralgo (2008) le compara con Tom Sawyer, héroe de la época gracias a la novela famosa de Mark Twain, por su afán de búsqueda de aventuras y porque a ambos les gustaban otras cosas que aquellas que ocurrían dentro de las aulas. Cajal cuenta con verdadera fruición los líos en los que se metía en busca de aventuras, como la voladura de la puerta a un vecino, o cómo robó unas rosas o cómo estuvo a punto de morirse al caerse a una balsa helada en pleno invierno. Sus verdaderas pasiones eran el dibujo, la lectura y la aventura. Las de su padre el estudio y el hacerse un hombre de provecho. En esta dialéctica transcurrió su infancia.

A los diez años, cuando se empezaba entonces el bachillerato, su padre lo llevó a estudiar al colegio de los escolapios de Jaca (Huesca), alojándose en casa de su tío Juan, hermano de su madre. Juan le trataba bien, pero su situación económica pasaba por un bache importante y por tanto en aquella casa se pasaba un hambre considerable por falta de recursos.

Al llegar hizo su examen de ingreso, que pasó brillantemente. Allí no tuvo problemas con sus compañeros, pero sí con las materias y el sistema de enseñanza y sobre todo con un padre Jacinto que decidió enseñarle latín y gramática costase lo que costase. El desinterés del niño Santiago por ambas materias era total, y los castigos a los que fue sometido por el padre. Jacinto rayaron en el maltrato. Pero en esa época disfrutó, en cambio, del apoyo de sus compañeros, que trataron de suavizar la situación de encierros y ayunos a los que era frecuentemente sometido para castigar su falta de interés por el estudio.

Los problemas que tenía Santiago con el método de los escolapios y las malas notas que obtenía, indujeron a D. Justo a trasladar a su hijo, a mitad de curso, al Instituto de Huesca (1864), cuando éste ya tenía 12 años. D. Justo que había sido profesor de su hijo pensaba que éste podía rendir mucho más y que algo no marchaba en su educación.

El cambio produjo un gran regocijo en Cajal, que quedó deslumbrado por la ciudad, acostumbrado hasta entonces a vivir en pueblos, y por la calidad de los profesores del Instituto. Sin embargo, allí se complicaron las cosas de nuevo, puesto que apareció otra vez el acoso, ahora mucho más grave que el primero:

"Entre los que más abusaban de sus fuerzas para conmigo, recuerdo a un tal Azcón...pigre crónico que habia interrumpido varias veces sus estudios. Frisaría en los 
diez y ocho o diez y nueve años" (Ramón y Cajal, 1923, p., 57).

Cuenta Cajal minuciosamente los motivos por los cuales era insultado, vejado y vapuleado por sus compañeros del instituto. Los insultos se pueden resumir en dos: "italiano y carne de cabra" (Ramón y Cajal, 1923, p. 58). El primero se debía a que se había criado en Ayerbe y este pueblo era famoso por sus muchos rebaños de cabras. El segundo le venía del hecho de vestir un abrigo largo que su madre le había confeccionado, contando con que iba crecer. Parece, además que en aquella época había algunos italianos con levita que recorrían los pueblos con una lira haciendo recitaciones poéticas.

Pero además de insultos había palizas contundentes, pues la actitud beligerante que ante los insultos tenía, en aquel entonces el niño Santiago, hacía que la conducta de Azcón y sus secuaces fuese más que enérgica. Lo cuenta así:

"Lancéme como un tigre sobre Azcón y sus insolentes amigos, repartiendo a diestro y siniestro puñetazos y puntapiés...repuestos de la sorpresa, los agredidos devolviéronme con creces la agresión, propinándome monumental paliza ... y así sucesivamente durante dos o tres meses (Ramón y Cajal, 1923, p. 58)”.

Cuando por fin se cansó de recibir golpes, se tranquilizó, desistió de enfrentarse y alcanzó la calma suficiente para poder pensar sobre cómo resolver el problema. De nuevo asombra la capacidad de análisis y la toma de decisiones llevada a cabo por aquel niño de tan sólo doce años. Después de analizar las cosas, decidió que tenía delante tres posibles actitudes:

"el halago y la lisonja hacia los atropelladores, la invocación a la autoridad de los superiores, o, en fin, el ejercicio supraintensivo de los músculos, combinado con la astucia” (Ramón y Cajal, 1923, p. 59).

Decidió que las dos primeras -- halago y acusación -eran deshonrosas, así que no le quedó disponible más que la tercera vía: el ejercicio de los músculos y la astucia. A ello se puso con ardor recordando una frase de su padre:
"Si quieres triunfar en las arduas empresas, pon en ellas toda tu voluntad" (Ramón y Cajal, 1923, p. 59).

Y comenzó su plan logrando dos cosas positivas: fortalecerse y alejarse de sus enemigos:

"resolví entregarme sistemáticamente a los ejercicios fisicos, a cuyo fin me pasaba solitario horas y horas, en los sotos y arboledas del Isuela, ocupado en trepar a los árboles, saltar acequias, levantar a pulso pesados guijarros, ejecutando, en fin, cuantos actos creía conducentes a acelerar mi desarrollo muscular, elevándolo al vigor máximo compatible con mis pocos años...el fruto de mi entrenamiento, como ahora se dice, fue magnifico. Desde el tercer curso (13 años), mis puños y la habilidad en el manejo de la honda y del palo infundieron respeto a los matones de los últimos años y hasta el atlético Azcón" (Ramón y Cajal, 1923, p. 59).

Esta nueva fortaleza corporal y su habilidad hondística le permitieron tener aplomo y seguridad para ir a advertir a Azcón que podía acertarle fácil y limpiamente con su honda sin acercarse siquiera, y dejarle bien tocado. Éste le tomo en serio, probablemente porque habría observado sus nuevas habilidades, y desistió en ese punto de sus insultos y bravuconerías, llegando a aceptarle, a admirarle e incluso a ser su amigo.

\section{Discusión y conclusiones}

En estas páginas estamos inequívocamente ante un caso de "acoso escolar", puesto que en él se dan todas las características exigidas para calificarlo de tal (agresión física y verbal repetida, perpetrada por individuos mayores en edad y número). En efecto, era algo que se hacía en grupo, en situaciones de ausencia de adultos, por niños mayores que el agredido, y se le ofendía con insultos y agresiones físicas, y todo ello, además, de forma reiterada. Están, pues, presentes todas las condiciones exigidas por los expertos para un caso de este tipo.

Por otro lado, la respuesta de Cajal es de naturaleza muy interesante y especial, que podría incluso conside- 
rarse como un ejemplo en su género. Nos conviene analizarlo, no sólo porque se trata de un niño muy particular, sino porque tanto sus soluciones como sus reflexiones pueden llegar a ser una inspiración para quienes se ocupan de estos temas, tanto desde el punto de vista de su prevención primaria como secundaria.

Las circunstancias de los acosos descritos se parecen a las actuales. Los niños acosados hoy y entonces se caracterizan por presentar características diferentes a la mayoría del conjunto de escolares que les rodean. Cajal llega a una escuela nueva, y no sólo no es aceptado, sino que es brutalmente agredido simplemente por ser distinto. En el caso de Cajal las diferencias, curiosamente eran positivas: él hablaba mejor, era listo, sabía muchas cosas, tenía muchas habilidades. Pero era "distinto", distinto por su habla y su vestido. Sus hábitos eran 'diferentes' y como se acostumbra a decir, eran por tanto 'indecentes'. La no aceptación de lo otro y la posible amenaza que esto puede suponer a un grupo es suficiente para generar un ataque en toda regla.

Lo aleccionador y sorprendente de este caso es que un niño muy pequeño pudo generar en solitario un sistema completo de defensa para la solución de su problema. Es verdad que no era un niño cualquiera, y por tanto no se puede sin más generalizar, ni exigir que todos los niños tengan esta misma capacidad de respuesta, pero sí se puede extraer y analizar sus soluciones para que puedan ser conocidas y usadas por otras personas preocupadas con el tema.

La primera de las soluciones que Cajal puso en movimiento en Ayerbe fue la asimilación: buscó hacerse invisible mediante la homogenización.

'Yo puedo hacer como ellos, para no parecer distinto y ser aceptado'. Esto es lo que parece haber pensado el primer Cajal de los ocho años. Y efectivamente fue una solución exitosa. Por tanto, podríamos pensar que éste puede ser un consejo útil a dar a niños envueltos en casos de acoso: limar las diferencias respecto del grupo. Y eso es lo que suelen hacer todos los niños inteligentes motu proprio, precisamente la adaptación por mimetismo, que consiste exactamente en eso.
Pero vemos también que la segunda solución, arbitrada por Cajal en el instituto de Huesca, cuando tenía ya doce años fue completamente distinta. En el primer caso era un niño y la solución fue la adaptación mimética, pero la segunda vez era ya un adolescente y en este caso la solución al acoso escogida fue distinta: el enfrentamiento. Esta solución, al comienzo, carente de precisión y de estrategia, fue un fracaso, pero sin embargo ello le llevó a un nuevo planteamiento y análisis del problema. Es importante ver el carácter reflexivo y diferido de su segunda respuesta, que le condujo al éxito. Fue necesario que en el proceso mediara una preparación física (para fortalecerse), la soledad (para evitar ser agredido) y además, la disciplina y la constancia para lograr la consecución de sus metas.

Esta evolución en la búsqueda de soluciones, hecha por un niño sumamente inteligente, también tiene que hacernos pensar que no todas las soluciones tienen que ser las mismas en los distintos períodos de edad. Los niños cambian, y su autoestima tiene diferentes goznes, y sobre todo en la adolescencia es importante que su conducta esté acorde con sus valores, precisamente para no perder la autoestima. Cajal (1940) decía a los científicos de su época: "vale el que sabe y actúa y no el que sabe y se duerme".

Y esto se lo aplicó antes a sí mismo, cuando tuvo que resolver los ataques de sus compañeros (Ramón y Cajal, 1940).

A propósito de todo ello, y ya desde su propia reflexión adulta, formuló D. Santiago una meditada valoración:

"Todos estos conflictos infantiles, que a muchos parecerán puras chiquilladas, tienen decisiva importancia, no solo para la formación del carácter, sino hasta para la conducta ulterior durante la edad viril" (Ramón y Cajal, 1940, p. 59).

Es evidente que este caso de acoso y su evolución es una fuente de ideas en la solución del grave problema que el acoso tiene en nuestros días. El caso de Ramón y Cajal es una fuente de inspiración en la búsqueda de soluciones de este grave problema. Sin duda ello se debe a que el 
caso fue analizado por una mente privilegiada, con experiencia del tema, de la cual deberíamos aprender a buscarle soluciones.

\section{Referencias}

Comunidad de Madrid (2016). Informe anual de acoso escolar de la Comunidad de Madrid. Recuperado de https://www.ecmadrid.org/es/pdfsrevistas/doc_download/4862-i-informe-anual-sobreacoso-escolar-cm-observatorio-de-la-convivencia$15-16$

Defensor del Pueblo (2000). Violencia escolar y maltrato entre iguales en la educación secundaria obligatoria. Madrid, España: Defensor del pueblo.

Defensor del Pueblo (2007). Violencia escolar y maltrato entre iguales en la educación secundaria obligatoria 1999-2006. Madrid, España: Defensor del pueblo.

Diaz-Aguado, M. J. (2006). Del acoso escolar a la cooperación en las aulas. Madrid, España: PearsonPrentice Hall.

Fundación Anar (2016). Violencia escolar. Madrid, España: Fundación Anar.

Garagordobil, M. y Oñederra, J. (2010) Violencia entre iguales. Madrid, España: Pirámide.

Garaigordobil, M., Martinez, V. y Alirin, J. (2015). Autoestima, empatía y conducta agresiva en adolescentes victimas de Bulling presencial. European Journal of Investigation in Health, Psychology and Education, 3, 29-40. http://dx.doi.org/10.1989/ejihpe.v3i1.21

Laín Entralgo, P. (2008). Escritos sobre Cajal. Madrid, España: Triacastela.
López Piñero, J. M. (1985). Cajal. Barcelona, España: Salvat.

Olweus, D. y Hodgins, S. (1993). Bulling/victim Problems among Scholars' Children: Long term Consequences and Effective Prevention Programs. In Mental Disorders and Crime (pp. 317-349). Thousand Oaks: Sage.

Tremblay, R. y Nagin, D. S., (2005). The developmental origins of physical aggression. New York: Guilford Press.

Ramón y Cajal, S. (1923). Recuerdos de mi vida. Madrid, España: Juan Pueyo.

Ramón y Cajal, S. (1894). Consideraciones generales sobre la morfología de la célula nerviosa. La Veterinaria Española, 37(1320), 257-260.

Ramón y Cajal, S. (1922). Charlas de café. Madrid, España: Espasa Calpe.

Ramón y Cajal, S. (1940). Reglas y consejos sobre la investigación científica (Los tónicos de la voluntad). Madrid, España: Librería Beltran. 